\title{
Fatigue, depression, and social adjustment in chronic fatigue syndrome
}

\author{
G A Walford, W McC Nelson, D R McCluskey
}

\begin{abstract}
The aims of this study were to determine the characteristics and perceived levels of fatigue and the prevalence of depression in children with chronic fatigue syndrome and to assess the effects of illness on schooling and social functioning. Twelve children with chronic fatigue syndrome were compared with a matched group of children with cystic fibrosis and matched healthy controls. Levels of fatigue (fatigue questionnaire), depression (children's depression inventory), and social adjustment (semistructured interview with parents) were compared between groups. Children with chronic fatigue syndrome had significantly higher median scores for physical and mental fatigue and depressive symptomatology than either comparison group and five children scored as depressed on the children's depression inventory. Schooling and social functioning were seriously disrupted.

Children with chronic fatigue syndrome reported high levels of fatigue affecting both physical and mental functioning, the association with depression found in adult studies was confirmed, and social adjustment was poor. (Arch Dis Child 1993; 68: 384-388)
\end{abstract}

Chronic fatigue syndrome or as it is often known, myalgic encephalomyelitis, remains a topic of interest and disagreement in the media and among doctors. A cardinal feature of the syndrome is a definite and identifiable onset of severe and disabling fatigue that affects both physical and mental functioning and that lasts for at least six months. A number of other symptoms are usually also present, in particular myalgia, sleep disturbance, and disturbance of mood. Significant physical signs and/or abnormal laboratory investigations are rare, and this has contributed to the ongoing debate as to the organic versus psychological nature of the syndrome.

Although the syndrome is said to be rare in children, ' child psychiatrists and paediatricians are referred small but increasing numbers of children for whom a diagnosis of chronic fatigue syndrome has been made, often by one or both parents. The implications for a child with this diagnosis may be considerable. The illness may run a variable and protracted course and the potential for disruption of schooling and normal peer interactions is a cause for concern. ${ }^{23}$

Studies of adults with chronic fatigue syndrome describe high levels of subjective fatigue affecting both physical and mental functioning ${ }^{+5}$ and high rates of depression, between $21 \%$ and $67 \%{ }^{+7}$

Despite the extensive literature on chronic fatigue syndrome among adult populations, there is little systematic research on children. ${ }^{3}$ We were interested to know whether a population of children with this disorder would also report high levels of perceived fatigue and whether rates of depression would be similar. If so, would these findings be specific for children with chronic fatigue syndrome or reported with similar frequency in children with other severe chronic and sometimes fatiguing illnesses such as cystic fibrosis.

\section{Patients and methods}

We studied 12 children attending a regional immunology clinic who had a diagnosis of chronic fatigue syndrome and assessed perceived levels of fatigue, associated symptoms, and mood state. We also looked at the effect of the illness on social relationships and schooling. To control for the effects of having a chronic, serious illness we compared the study group with a control group of children with cystic fibrosis. A further control group of healthy children from a similar environment and growing up in Northern Ireland were also compared with the study group.

\section{STUDY CHILDREN}

The 12 study children, aged 16 and under, were selected consecutively over a nine month study period and they fulfilled the following criteria.

(1) New onset of severe, disabling fatigue of at least six months' duration that was severe enough to reduce or impair daily activity by at least $50 \%$ of the premorbid level.

(2) The presence of additional symptoms such as emotional disturbance, myalgia, and sleep

\section{Table 1 Chronic fatigue syndrome}

(A) A syndrome characterised by fatigue as the principal symptom

(B) A syndrome of definite onset that is not life long

(C) The fatigue is severe, disabling, and affects physical and mental functioning

(D) The symptom of fatigue should have been present for a minimum of six months during which it was present for more than $50 \%$ of the time

(E) Other symptoms may be present, particularly mvalgia, mood, and sleep disturbance

(F) Certain patients should be excluded from the definition. They include:

They include: (i) Patients with established medical conditions known to
produce chronic fatigue (for example, severe anaemia). produce chronic fatigue (for example, severe anaemia). condition is diagnosed at presentation or only condition is diagnosed at presentation or only
subsequently. All patients should have a history and subsequently. All patients should have a history and
physical examination performed by a competent physician ii) Patients with a current diagnosis of schizophrenia, manic (ii) Patients with a current diagnosis of schizophrenia, man
depressive illness, substance abuse, eating disorder, or proved organic brain disease. Other psychiatric disorders (including depressive illness, anxiety disorders, and hyperventilation syndrome) are not necessarily reasons for exclusion 
disturbance, which are characteristic features of the disorder in adults.

(3) Exclusion, by careful history, physical examination, and relevant laboratory tests, or other medical conditions that could account for the symptoms. ${ }^{8}$

All children also fulfilled criteria for a more recently proposed definition of chronic fatigue syndrome shown in table $1 .^{9}$

All eligible children and their parents agreed to take part in the study.

\section{PATIENT COMPARISON GROUP}

Twelve children, matched for age and sex, were selected consecutively from a population of children attending a regional cystic fibrosis clinic. It was not possible to match these children for social class because of the relative lack of children from higher socioeconomic groups.

\section{NORMAL SUBJECTS}

Twelve healthy children, matched for age, sex, and social class, were selected from the general population. All 12 children were related to or were acquaintances of hospital staff. These children were considered to be in good health and not suffering from any illness or complaint that had required attendance at medical, psychiatry, or psychology clinics during the preceding 12 months.

\section{ASSESSMENTS}

All children were assessed by a child psychiatrist using:

\section{(1) Fatigue questionnaire}

A questionnaire developed for use in adults with chronic fatigue ${ }^{+10}$ was modified by the authors for use with children in this study. It comprises three sections, one to assess perceived levels of fatigue, one concerned with associated symptoms, and a third to assess attribution of symptoms to physical or psychological causes and patient safisfaction with treatment from doctors. The third section was not completed by the healthy comparison group.

The section on fatigue includes 13 items recording subjectively aspects of fatigue that the authors ${ }^{+}$have arbitrarily divided into eight physical and five mental complaints (table 2). This was later confirmed using principal components analysis. Each item is scored 0,1 , or 2 corresponding to same as usual, worse than usual, and much worse than usual. Results are then listed under physical or mental symptoms giving a maximum score of 16 for physical and 10 for mental fatigue.

\section{(2) Children's depression inventory"}

This is a 27 item self report questionnaire developed from the Beck depression inventory for adults. ${ }^{12}$ It has been well validated and normative data are available. ${ }^{13}$ Each item consists of three statements of increasing severity scored 0,1 , or 2 in the direction of increasingly depressed mood. The maximum obtainable score is 54 .
Table 2 Fatigue symptoms assessed

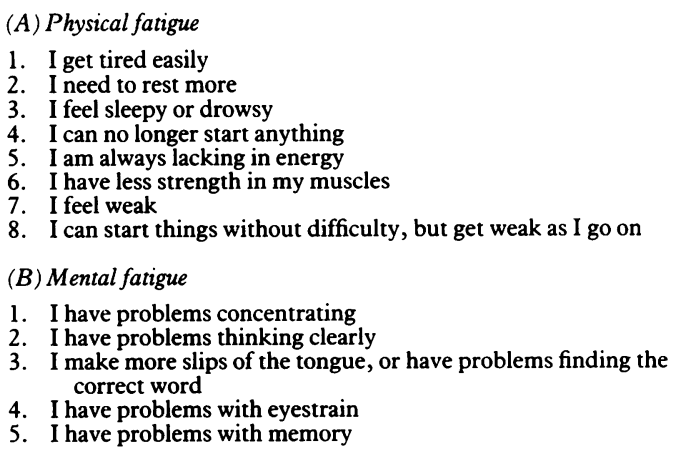

A score of 19 or more was taken as a cut off for depressed mood in children. ${ }^{1+}$

\section{(3) Assessment of social adjustment}

This is a semistructured interview with one or both parents to assess social functioning and school attendance.

\section{ANALYSIS}

Scores on the fatigue questionnaire and depression inventory were analysed using the Wilcoxon matched pairs signed ranks test.

\section{Results}

\section{DEMOGRAPHIC DATA}

There were six boys and six girls with chronic fatigue syndrome. Five children were referred by their general practitioner directly to the immunology clinic. Four were initially referred to paediatricians, one to a surgeon, and two to child psychiatry, one of whom was initially misdiagnosed as a case of anorexia nervosa. In three cases the mother initially made the diagnosis. Before their current illness, no child had had a referral to child psychiatry or psychology, although one child had a history of reluctance to attend school.

The mean duration of illness was 2.9 years (range $0 \cdot 8-6 \cdot 0$ years). There was an overrepresentation of the higher socioeconomic groups with eight children from social classes I and II and four children from social class III. ${ }^{15}$ Two of the children were siblings and in a further two cases a member of the child's immediate family had had or was currently suffering with chronic fatigue syndrome. All 12 children described an acute infectious illness immediately before or at the onset of symptoms.

There was no significant difference in the mean ages 13.9 years, 13.6 years, and 13.9 years for children with chronic fatigue syndrome, cystic fibrosis, and healthy children respectively and the age range 8-16 years was the same for all three groups.

\section{FATIGUE QUESTIONNAIRE}

Children with chronic fatigue syndrome admitted to significantly higher levels of physical (median 9.5) and mental (median 4.0) fatigue 
Table 3 Results of fatigue questionnaire (physical and mental fatigue scores) and children's depression inventory. Results are median (95\% confidence interval)

\begin{tabular}{|c|c|c|c|}
\hline & $\begin{array}{l}\text { Chronic fatigue } \\
\text { syndrome }\end{array}$ & Cystic fibrosis & Healthy controls \\
\hline $\begin{array}{l}\text { Physical fatigue } \\
\text { Mental fatigue } \\
\text { Children's depression inventory }\end{array}$ & $\begin{array}{c}9 \cdot 5(7 \cdot 0 \text { to } 12 \cdot 5) \\
4 \cdot 0(3 \cdot 0 \text { to } 5 \cdot 5) \\
13 \cdot 8(7 \cdot 0 \text { to } 20 \cdot 0)\end{array}$ & $\begin{array}{l}2 \cdot 0^{\star \star}(0.5 \text { to } 4.5) \\
0 \cdot 0^{\star \star}(0.0 \text { to } 1.5) \\
6 \cdot 0^{\star}(3.0 \text { to } 10.0)\end{array}$ & $\begin{array}{l}0 \cdot 0^{\star \star}(0.0 \text { to } 0.5) \\
0.5^{\star \star}(0.0 \text { to } 1 \cdot 5) \\
5 \cdot 0^{\star}(2 \cdot 0 \text { to } 10.5)\end{array}$ \\
\hline
\end{tabular}

Wilcoxon matched pair signed ranks test: ${ }^{\star} \mathrm{p} \leq 0.05,{ }^{\star \star} \mathrm{p} \leq 0.01$.

than children with cystic fibrosis who had median scores of 2.0 for physical and 0.0 for mental fatigue $(\mathrm{p}<0 \cdot 01)$. Normal subjects also had significantly lower scores for physical (median 0.0 ) and mental (median 0.5 ) fatigue than children with chronic fatigue syndrome $(\mathrm{p} \leq 0.01)$.

Two children with cystic fibrosis scored 7 or more for physical fatigue and had no accompanying mental fatigue and one child in the healthy control group had high scores, similar to those of the study group, for physical and mental fatigue.

All children with chronic fatigue syndrome felt that their fatigue had affected their ability to walk long distances and during the worst stages of illness one child was bedridden for several months and five other children were severely immobilised and felt unable to walk further than a few feet within or between rooms. Two of these children had requested wheelchairs. In contrast, all children in both comparison groups felt able to walk further than one mile; this included the children with cystic fibrosis who had reported high levels of physical fatigue and the healthy comparison case with high perceived levels of physical and mental fatigue.

\section{ADDITIONAL SYMPTOMS}

Neuropsychological symptoms are often found in association with chronic fatigue syndrome and 11 children in the study group complained of impairment of concentration and/or memory (concentration 11, memory seven). This was in contrast to only two children with cystic fibrosis reporting impaired concentration, and none reporting memory problems. Two children in the healthy comparison groups reported impairment of concentration and one of these also had memory impairment.

On the associated symptom checklist section of the fatigue questionnaire children with chronic fatigue syndrome admitted to a mean number of 8.3 somatic symptoms. The most frequently complained of were sleep disturbance $(\mathrm{n}=12$, difficulty in getting to sleep 10 , daytime drowsiness 7$)$, frequent headaches $(n=11)$, myalgia $(n=11$, at rest and on exercise 9 , on exercise alone 2$)$, stomach pains $(n=8)$, eyestrain $(n=7)$, pain in eyes $(n=7)$, increased sensitivity to light $(n=7)$.

Children with cystic fibrosis reported a mean number of 4.5 somatic symptoms, in particular gastrointestinal and respiratory problems and the majority of these were attributable to cystic fibrosis. Only three children complained of sleep disturbance and in one of these the symptoms were attributable to chest infections. Two reported frequent headaches and one complained of muscle pains on exercise.
The mean number of somatic symptoms admitted to by healthy children was $2 \cdot 1$. Four reported sleep disturbance (difficulty in getting to sleep 2, daytime drowsiness 2), four complained of frequent headaches, and two of muscle pains, one on exercise, the other at rest and in exercise. One child in the healthy comparison group admitted to 14 somatic symptoms.

\section{CHILDREN'S DEPRESSION INVENTORY}

Depressed patients often complain of excessive fatigue and hence many depression inventories include an item on tiredness. In order to avoid undue circularity when investigating symptoms of depression in children with chronic fatigue syndrome, their depression scores were adjusted to exclude the score on the item for tiredness. Adjusted scores are given in table 3.

Children with chronic fatigue syndrome had a significantly higher median score for depression $(13.8)$ than either children with cystic fibrosis (median 6.0, $\mathrm{p}<0.05$ ) or healthy controls (median 5.0, $\mathrm{p}=0.05$ ).

Five children with chronic fatigue syndrome scored 19 or over (range 19-30) suggesting significant depressive symptomatology. Three of these five children were taking antidepressant medication that they and their parents perceived as having a beneficial effect on their mood. None of the children who scored below 19 were taking antidepressants.

The highest score in the cystic fibrosis group was 12 . Among the healthy control subjects one case, a female aged 16 years, scored 20 on the depression inventory. She also obtained a high score on the fatigue questionnaire and had admitted to 14 somatic symptoms.

\section{SOCIAL ADJUSTMENT AND SCHOOLING}

During the worst phase of the illness, all 12 children with chronic fatigue syndrome had been absent from school for between one and eight complete school terms and four of these children had missed five or more complete terms. At the time of their interview nine children were attending part time (in some cases as little as one day a week). Five children had had to repeat at least one year's schooling. Eleven children felt that their condition had affected their ability to meet and make friends and their parents confirmed this.

In contrast, all children with cystic fibrosis were attending school regularly and the only prolonged absences were for hospital admissions for treatment of chest infections lasting between one and five weeks. No child missed more than eight weeks of school in any one academic year and most missed far less than this. No child in this group had had to repeat a year's schooling and none of these children felt that their illness had affected their peer relationships adversely.

\section{SATISFACTION WITH TREATMENT AND} ATTRIBUTION OF SYMPTOMS

Four children with chronic fatigue syndrome felt unhappy at the way they had been treated. Three 
of these also felt that doctors had treated them unpleasantly some or all of the time.

Six children often or always felt that doctors hadn't believed them. Six children felt that their illness had a physical cause and that there was no psychological component to their symptoms at all.

\section{Discussion}

DEMOGRAPHIC DETAILS

Although our numbers are small, the bias towards higher socioeconomic groups among children with chronic fatigue syndrome is similar to findings in adult populations. ${ }^{16}$ The fact that the diagnosis of chronic fatigue syndrome is often made or suggested by a parent has previously been reported. ${ }^{2}$

\section{FATIGUE}

The characteristics and levels of fatigue complained of by the study group are similar to findings in an adult population ${ }^{4}$ with all cases admitting to fatigue affecting mental as well as physical functioning and precipitated by mental as well as physical effort. The slightly lower levels of fatigue admitted to by some children may reflect the degree of recovery they and their parents reported. There has been considerable debate as to whether the fatigue itself has its origin centrally in the brain or peripherally at or beyond the neuromuscular junction. Arising from this, attempts have been made to differentiate between symptoms of mental fatigue, which are presumed to originate centrally, and those of physical fatigue, which may be either central or peripheral in origin. Using this arbitrary division of symptoms of fatigue into mental and physical aspects, Wessely and Powell found that a group of patients with unexplained chronic fatigue complained of both mental and physical fatigue in contrast to controls with neuromuscular disorders who complained only of physical fatigue unless they had a coexisting psychiatric disorder and in these cases symptoms of mental fatigue were also present. ${ }^{4} \mathrm{~A}$ further comparison group of depressed psychiatric patients reported similarly high levels of physical and mental fatigue as the patients with chronic fatigue. These results are similar to those of Wood et al who compared a group of patients with chronic fatigue with a group with muscle disease. ${ }^{5}$ Both groups complained of physical weakness but whereas high levels of 'troublesome mental fatigue' were reported by most chronic fatigue sufferers, only mild levels of mental fatigue were admitted to by the group with muscle disease. In the present study all children with chronic fatigue syndrome complained of mental as well as physical fatigue, suggesting that central mechanisms were involved in the illness.

\section{ADDITIONAL SYMPTOMS}

Children with chronic fatigue syndrome admitted to a number of additional symptoms of a similar type and with a similar frequency as those reported in adult populations of fatigue suf- ferers. ${ }^{17}$ Sleep disturbance (100\%), myalgia $(92 \%)$, and headaches (92\%) were particularly common. These symptoms were in themselves non-specific and were also complained of by small numbers of children in both comparison groups.

It is possible that the number and patterns of symptoms admitted may be more helpful in making a diagnosis than the presence or otherwise of individual symptoms sometimes held to be in some way specific to chronic fatigue syndrome.

\section{DEPRESSION}

Children with chronic fatigue syndrome had significantly more depressive symptomatology than either of the control groups. One possibility for this is that depressed mood might simply be a reaction to chronic physical illness but the comparatively low levels of depressive symptoms in children with cystic fibrosis does not support this. Five children $(42 \%)$ with chronic fatigue syndrome scored as depressed on the children's depression inventory and this is consistent with the commonly found association between chronic fatigue syndrome and depression reported in adult studies. ${ }^{471819}$ One hypothesis is that chronic fatigue syndrome may be a misdiagnosed depressive disorder but the findings in other studies as well as our own, of a number of patients who are not depressed, do not support this view. 'Depression is a sufficient but not necessary explanation' .

The finding of one child in the healthy control group who reported considerable physical and mental fatigue and depressive symptomatology deserves mention. She also complained of myalgia, sleep disturbance, and headaches. In many ways her symptoms were very similar to those of the children with chronic fatigue syndrome, although they were not impairing her daily activity to the same extent; she was still attending school regularly and could walk long distances (a mile or more). She was from a professional family and was highly motivated to achieve well academically. Initially it was thought that some of the symptomatology was related to frustration and anxiety with schoolwork. Her symptoms persisted in the school holidays and she began to diet excessively. A diagnosis of anorexia nervosa was made by the consultant psychiatrist to whom she was referred.

\section{ATTRIBUTION OF SYMPTOMS}

In studies of adults attribution of symptoms to a physical cause is common, ${ }^{4}$ and a strong conviction that their illness had a physical cause was common in children in this study.

It has been suggested that attribution of psychological symptoms to an organic cause that no longer applies may, at least initially, serve a protective function reducing the levels of stress experienced by a patient but that in the longer term such beliefs may lead to more extensive and seriously disabling illness behaviour..$^{20}$ The three children in this study who had the longest duration of illness (four years or more) all 
attributed their illness to entirely physical factors.

We were concerned at the dissatisfaction with treatment expressed by some children and from the accounts the children and their parents gave, it appeared that much of the perceived unpleasantness from doctors was related to disagreement between doctors, patients, and parents as to the organic or psychological nature of their symptoms.

Although there is no one proved effective treatment for chronic fatigue syndrome to date, some promising treatment and management strategies have been described recently. ${ }^{32122}$ These are unlikely to be implemented if relations between physician, child, and parents are hampered by what many perceive as fruitless disagreement concerning the physical or psychological aetiology of the illness.

In summary, the clinical picture of chronic fatigue syndrome in children in this study is similar to that described in adults with high levels of subjective fatigue affecting both physical and mental functioning. The commonly found association between chronic fatigue syndrome and depression reported in adult studies was confirmed for children in this study and the comparatively low levels of depressive symptomatology reported by children with cystic fibrosis suggest that this is not simply an effect of having a chronic disabling illness.

The consequences of the disease for many children in terms of missed schooling and reduced social interactions are considerable and often occur at a crucial time in a young person's development through late childhood and adolescence. This is a cause for concern and an important consideration when treatment and management strategies are planned.

The authors gratefully acknowledge the valuable contribution of the following: Dr S Wessely, Dr F Leddy, Dr A Redmond, Dr C Patterson.

1 David AS, Wessely S, Pelosi AJ. Postviral fatigue syndrome: time for a new approach. $B M \mathcal{F} 1988$; 296: 696-9.

2 Harris F, Taitz $L$. Damaging diagnoses of myalgic encephalitis in children. BMF 1989; 299: 790 .

3 Vereker MI. Chronic fatigue syndrome: a joint paediatricpsychiatric approach. Arch Dis Child 1992; 67: 550-5.

4 Wessely S, Powell R. Fatigue syndromes: a comparison of chronic 'postviral' fatigue with neuromuscular and affective disorders. F Neurol Neurosurg Psychiatry 1989; 52: 940-8.

5 Wood GC, Bentall RP, Gopfert M, Edwards RHT. A comparative psychiatric assessment of patients with chronic fatigue syndrome and muscle disease. Psychol Med 1991; 21 : 619-28.

6 Taerk GS, Toner BB, Salit IE, Garfinkel PE, Ozersky S. Depression in patients with neuromyasthenia (benign myalgic encephalomyelitis). Int $\mathcal{F}$ Psychiatry Med 1987; 17: myalgic

7 Hickie I, Lloyd A, Wakefield D, Parker G. The psychiatric status of patients with chronic fatigue syndrome. $\mathrm{Br} \mathcal{F}$ Psychiatry 1990; 156: $534-40$.

8 McBride SJ, McCluskey DR. Treatment of chronic fatigue syndrome. BrMed Bull 1991; 47: 895-907.

9 Sharpe MC, Archard LC, Banatvala JE, et al. A report chronic fatigue syndrome: guidelines for research. $\mathcal{J} R$ Soc Med 1991; 84: 118-21.

10 Butler S, Chalder T, Ron M, Wessely S. Cognitive behaviour therapy in chronic fatigue syndrome. $\mathcal{F}$ Neurol Neurosurg Psychiatry 1991; 54: 153-8.

11 Kovacs M. The children's depression inventory. Psychopharmacol Bull 1985; 21: 995-8.

12 Beck A, Ward C, Mendelson M, Mock J, Erbaugh J. An inventory for measuring depression. Arch Gen Psychiatry inventory for meas

13 Smucker MR, Craighead WE, Craighead LW, Green BJ. Normative and reliability data for the children's depression Normative and reliability data for the children's depress
inventory. $\mathcal{F}$ A bnormal Child Psychol 1986; 14: 25-39.

14 Knight D, Hensley VR, Waters B. Validation of the children's nnight $\mathrm{D}$, Hensley VR, Waters $\mathrm{B}$. Validation of the children's
depression inventory in a prepubertal sample. $\mathcal{F}$ Child Psychol Psychiatry 1988; 29: 853-63.

15 Office of Population Censuses and Surveys. Classification of occupations. London: HMSO, 1980.

16 Lewis G, Wessely S. The epidemiology: of fatigue: more questions than answers. $\mathcal{f}$ Epidemiol Community Health 1992; 46: 92-7.

17 Dowsett EG, Ramsay AM, McCartney R.A, Bell EJ. Mvalgic encephalomyelitis - a persistent enteroviral infection? Postgrad Med 7 1990; 66: 526-30.

18 Ray C. Chronic fatigue syndrome and depression: conceptual and methodological ambiguities. Psychol Med 1991; 21: 1-9.

David AS. Postviral fatigue syndrome and psychiatry. $\mathrm{Br}$.Med Bull 1991; 47: 966-88.

20 Woods TO, Goldberg DP. Psychiatric perspectives: an overview. Br Med Bull 1991; 47: 908-18.

21 Sharpe M. Psychiatric management of PVFS. Br . Med Bull 1991; 47: 989-1005.

22 Lask B, Dillon MJ. Postviral fatigue syndrome. Arch Dis Child 1990; 65: 11 . 